\title{
Association between dietary inflammatory index and prostate cancer among Italian men
}

\author{
Nitin Shivappa ${ }^{1,2}$, Cristina Bosetti ${ }^{3}$, Antonella Zucchetto ${ }^{4,5}$, Maurizio Montella ${ }^{6}$, Diego Serraino ${ }^{4}$, \\ Carlo La Vecchia ${ }^{5}$ and James R. Hébert ${ }^{1,2,7 *}$ \\ ${ }^{1}$ Cancer Prevention and Control Program, University of South Carolina, 915 Greene Street, Suite 241, Columbia, \\ SC 29208, USA \\ ${ }^{2}$ Department of Epidemiology and Biostatistics, Arnold School of Public Health, University of South Carolina, \\ Columbia, SC 29208, USA \\ ${ }^{3}$ Department of Epidemiology, IRCCS - Istituto di Ricerche Farmacologiche "Mario Negri", Milan, Italy \\ ${ }^{4}$ S.O.C. Epidemiologia e Biostatistica, Centro di Riferimento Oncologico, Aviano (PN), Italy \\ ${ }^{5}$ Department of Clinical Sciences and Community Health, Università degli Studi di Milano, Milan, Italy \\ ${ }^{6}$ Dipartimento di Epidemiologia, 'Fondazione G. Pascale', Istituto Nazionale Tumori, Naples, Italy \\ ${ }^{7}$ Department of Family and Preventive Medicine, University of South Carolina School of Medicine, Columbia, SC 29208, USA
}

(Submitted 26 March 2014 - Final revision received 11 September 2014 - Accepted 14 October 2014 - First published online 17 November 2014)

\begin{abstract}
Previous studies have shown that various dietary components may be implicated in the aetiology of prostate cancer, although the results remain equivocal. The possible relationship of inflammation derived from dietary exposures with prostate cancer risk has not been investigated. We examined the ability of a newly developed dietary inflammatory index (DII) to predict prostate cancer risk in a case-control study conducted in Italy between 1991 and 2002. A total of 1294 patients aged $<75$ years with incident, histologically confirmed carcinoma of the prostate served as cases. A total of 1451 subjects aged $<75$ years who were admitted to the same hospitals as cases for a wide spectrum of acute, non-neoplastic conditions served as controls. The DII was computed based on dietary intake assessed using a previously validated seventy-eight-item FFQ. Logistic regression models were used to estimate multivariable OR adjusted for age, study centre, years of education, social class, BMI, smoking status, family history of prostate cancer and total energy intake. Men with higher DII scores had a higher risk of prostate cancer when analysed using the DII as both continuous (OR 1.06, 95\% CI 1.00, 1.13) and categorical, i.e. compared with men in the lowest quartile of the DII, men in the third and fourth quartiles were at elevated risk (OR $\mathrm{Quartile}_{2 v .1} 1.32,95 \%$ CI $1.03,1.69$ and $\left.\mathrm{OR}_{\text {Quartile } 4 v .1} 1.33,95 \% \mathrm{CI} 1.01,1.76 ; P_{\text {trend }}=0.04\right)$. These data suggest that a pro-inflammatory diet, as indicated by the increasing DII score, is a risk factor of prostate cancer in Italian men.
\end{abstract}

Key words: Dietary inflammatory index: Diet: Inflammation: Prostate cancer: Case-control studies: Italy

Inflammation is an important factor contributing to cancer ${ }^{(1,2)}$, and considerable evidence for the role of chronic inflammation in prostate cancer is accumulating ${ }^{(3-5)}$. While inflammation typically occurs as part of the body response to tissue insult/injury ${ }^{(2,6)}$, chronic inflammation is a persistent condition in which tissue destruction and repair occur simultaneously $^{(7,8)}$. This involves the continuous recruitment of pro-inflammatory cytokines associated with increased blood flow to the injured tissue, due to histamine released by damaged mast cells ${ }^{(2)}$.

A recent case-control study has shown that the levels of C-reactive protein are higher in men with prostate cancer than in those with benign prostatic hypertrophy ${ }^{(9)}$, and the
Melbourne Collaborative Cohort Study has reported higher levels of IL-6, a pro-inflammatory cytokine, among malignant prostate cancer cases compared with those with benign prostate disease $\mathrm{e}^{(10)}$. These data are consistent with the hypothesis that innate immunity and inflammation play a role in prostate cancer $^{(11)}$.

Diet represents a complicated set of exposures that often interact and whose cumulative effect modifies both inflammatory responses and health outcomes. Although several studies have been conducted, the relationship between diet and prostate cancer is still unclear ${ }^{(12,13)}$. According to the Second Expert Report from the World Cancer Research Fund $^{(14)}$, foods containing lycopene and Se are protective

Abbreviation: DII, dietary inflammatory index.

*Corresponding author: Dr J. R. Hébert, fax +1 803576 5624, email jhebert@sc.edu 
against prostate cancer, while diets high in $\mathrm{Ca}$ increase its risk. A positive association between elevated intake of meat ${ }^{(12,15,16)}$ and milk and dairy products ${ }^{(17-20)}$ with the risk of prostate cancer has been observed. Conversely, an inverse association has been found for vegetable intake, but the results have been inconsistent $^{(21-24)}$. The possible relationship between inflammation derived from dietary exposure and the risk of prostate cancer has not been investigated.

The paucity of research related to diet and inflammation is probably due to logistic issues resulting from methodological complexity involved in linking diet, inflammation and cancers in the same study. In an effort to fill this methodological gap, researchers at the University of South Carolina's Cancer Prevention and Control Program developed a dietary inflammatory index (DII), which can be used in diverse populations in order to predict the levels of inflammatory markers and related health outcomes ${ }^{(25,26)}$. The development of the DII involved careful review and scoring of the scientific literature on diet and inflammation, and obtaining of datasets from around the world for comparison with dietary intakes of individuals ${ }^{(25)}$. Thus far, the DII has been found to be associated with inflammatory cytokines including C-reactive protein and IL- $6^{(26-28)}$, the glucose-intolerance component of the metabolic syndrome, increased odds of asthma and reduced forced expiratory volume in $1 \mathrm{~min}\left(\mathrm{FEV}_{1}\right)$, shift work and colorectal cancer (CRC) among women from the Iowa Women's Health Study ${ }^{(27-29)}$.

The purpose of the present study was to examine the association between the DII and the risk of prostate cancer in a case-control study conducted in Italy. A previous casecontrol study has revealed a positive association with the increased intake of milk and dairy products and a possible protective effect of vegetable intake ${ }^{(30)}$.

\section{Methods}

Full details of the case-control study have been published elsewhere $^{(30)}$. Briefly, the study was conducted between 1991 and 2002 in four Italian areas, including the greater Milan area and the provinces of Pordenone and Gorizia in northern Italy, the province of Latina in central Italy, and the urban area of Naples in southern Italy. Cases were 1294 patients aged $<75$ years (median age 66 years, range $46-74$ years) who were admitted to major teaching and general hospitals in the areas under study with incident, histologically confirmed carcinoma of the prostate, diagnosed no longer than 1 year before the interview. Controls were 1451 subjects aged $<75$ years (median age 63 years, range $46-74$ years) who were selected among patients admitted to the same hospitals as cases for a wide spectrum of acute, non-neoplastic conditions, not related to known or potential risk factors for prostate cancer and long-term modifications of diet. The main diagnostic categories of controls were traumatic conditions, mostly sprains and fractures (21\%); non-traumatic orthopaedic disorders, such as low back and disc disorders (33\%); acute surgical conditions, mostly abdominal such as appendicitis or strangulated hernia (17\%); and other illnesses, such as eye, ear, nose, skin and dental disorders (29\%).
Information on sociodemographic characteristics, anthropometric measures, lifestyle habits, including smoking status and alcohol drinking, personal medical history, and family history of cancer in first-degree relatives was assessed during subjects' hospital stay using a standard questionnaire that was administered by trained interviewers to the cases and controls. The subjects' usual diet during the 2 years before the diagnosis of cancer (or hospital admission, for controls) was assessed using an interviewer-administered FFQ, including seventy-eight foods and beverages, as well as a range of the most common Italian recipes. Subjects were asked to indicate the average weekly frequency of consumption of each dietary item; intakes lower than once per week, but at least once per month, were coded as 0.5 per week. Nutrient and total energy intake was determined using the Italian food composition database ${ }^{(31)}$. The FFQ showed satisfactory validity ${ }^{(32)}$ and reproducibility ${ }^{(33,34)}$ with Spearman's correlation coefficients between 0.50 and 0.60 for validity and between 0.60 and 0.70 for reproducibility.

Briefly, to calculate the DII for the subjects of the present study, the dietary data were first linked to the world database that provided a robust estimate of the mean and standard deviation for each food parameter considered ${ }^{(25)}$. This was achieved by subtracting the 'standard global mean' from the intake reported via the FFQ and dividing this value by the standard deviation (both calculated from the world database) in order to get $z$ scores. To minimise the effect of 'right skewing', these $z$ scores were then converted to a centred percentile score. The centred percentile score of each food parameter for each subject was then multiplied by the respective effect score of food parameters (inflammatory potential for each food parameter), which was derived from the literature review, in order to obtain a food parameter-specific DII score for a subject. All of the food parameter-specific DII scores were then summed to create the overall DII score for each subject in the study ${ }^{(25)}$. A description of the validation work, including both dietary recalls and a structured questionnaire similar to a FFQ, is also available ${ }^{(26)}$.

The food parameters used for the calculation of the DII were carbohydrate, protein, fat, alcohol, fibre, cholesterol, saturated fat, monounsaturated fat, polyunsaturated fat, $n-3$ PUFA, n-6 PUFA, niacin, thiamin, riboflavin, vitamin $\mathrm{B}_{6}$, Fe, Zn, vitamin A, vitamin $\mathrm{C}$, vitamin $\mathrm{D}$, vitamin $\mathrm{E}$, folic acid, $\beta$-carotene, anthocyanidins, flavan-3-ol, flavonol, flavonones, flavones, isoflavones, caffeine and tea. A higher DII score indicates a more pro-inflammatory diet and a lower DII score indicates a more anti-inflammatory diet. BMI was calculated as weight (in $\mathrm{kg}$ ) divided by height (in $\mathrm{m}$ ) squared, and was categorised into <24.0, 24-25.9, 26.0-28.9 and $\geq 29 \cdot 0 \mathrm{~kg} / \mathrm{m}^{2(35)}$.

\section{Statistical analyses}

The DII was analysed both as a continuous variable and by quartiles of exposure. The DII (as quartiles) was examined using the ANOVA test for continuous variables or the $\chi^{2}$ test for categorical ones across the following characteristics: age; 
years of education; BMI; smoking status; family history of prostate cancer. To understand the dietary profile of each quartile of the DII, we examined the distribution of various food groups across the quartiles of the DII. An ANOVA was used to test for the differences among the dietary groups. We estimated the OR and the corresponding 95\% CI using logistic regression models, adjusted for age, study centre, years of education, social class, BMI, smoking status, family history of prostate cancer, and total energy intake ${ }^{(36)}$. The covariates were chosen $a$ priori as they have been previously shown to be the risk factors of prostate cancer. Tests for linear trend were performed using the median value of each quartile as an ordinal variable. Statistical tests were performed using SAS ${ }^{\circledR} 9.3$ (SAS Institute, Inc.). All $P$ values were two-sided.

\section{Results}

The distribution of prostate cancer cases and controls according to age, years of education, and other selected variables is presented in Table 1 . The cases were somewhat older than the controls $(>50 v$. $\leq 50$ years: OR $2 \cdot 3,95 \%$ CI $1 \cdot 3$, $4 \cdot 3$ ), were more highly educated ( $\geq 12$ years of education $v$. $<7$ years of education: OR $1.9,95 \%$ CI $1.5,2 \cdot 3)$, had a higher social class (lower $v$. higher: OR 2.4, 95\% CI 1.7, 3.0), and more often reported a first-degree relative with prostate cancer (family history of prostate cancer $v$. no family history OR 3.9, 95\% CI 2.5, 5.8). No differences in the BMI level were observed between cases and controls. All factors were included in the analysis.

Small differences were observed in sociodemographic characteristics, anthropometric measures and lifestyle habits across the quartiles of the DII (data not shown). However, current smokers were more frequently in the higher quartiles of the DII than in the lower quartile, i.e. $32 \%$ of those in the highest quartile $v .26 \%$ of the controls in the lowest quartile. There were fewer men who were overweight or with a family history of prostate cancer in the higher quartiles of the DII.

Concerning the distribution of various food groups, there was a significant reduction in the consumption of vegetables, fruits, poultry and fish, and a significant increase in the consumption of pork, sugars, cheese and bread across the quartiles of the DII (Table 2). The OR and corresponding 95\% CI of prostate cancer according to the quartiles of the DII are shown in Table 3. In the age-adjusted models, no meaningful association was found between the DII and the risk of prostate cancer. However, in multivariable analysis, significant positive associations were found, with an OR of 1.06 (95\% CI 1.00, 1.13) for a one-unit increment in the DII (corresponding to approximately $7 \%$ of its global range) ${ }^{(25)}$. In the analysis using the DII expressed as quartiles, a significant trend of increasing risk $\left(P_{\text {trend }}=0.04\right)$ was found; however, there was some indication of flattening in the last two quartiles (OR $\mathrm{OR}_{\text {Quartile } 3 v .1} 1 \cdot 32,95 \% \mathrm{CI} 1.03,1.69$ and $\mathrm{OR}_{\text {Quartile } 4 v .1} 1.33,95 \%$ CI 1.01, 1.76) when compared with men in the lowest quartile of the DII.
Table 1. Distribution of 1294 cases of prostate cancer and 1451 controls according to age, years of education, and other selected covariates in the study conducted in Italy between 1991 and 2002

(Number of subjects and percentages)

\begin{tabular}{|c|c|c|c|c|}
\hline \multirow[b]{2}{*}{ Variables } & \multicolumn{2}{|c|}{ Cases } & \multicolumn{2}{|c|}{ Controls } \\
\hline & $n$ & $\%$ & $n$ & $\%$ \\
\hline \multicolumn{5}{|l|}{ Age (years) } \\
\hline$<60$ & 219 & $17 \cdot 0$ & 431 & $29 \cdot 6$ \\
\hline $60-64$ & 310 & $24 \cdot 0$ & 359 & $24 \cdot 7$ \\
\hline $65-69$ & 419 & $32 \cdot 4$ & 364 & $25 \cdot 1$ \\
\hline $70-74$ & 346 & $26 \cdot 7$ & 297 & $20 \cdot 5$ \\
\hline \multicolumn{5}{|l|}{ Education (years) ${ }^{\star}$} \\
\hline$<7$ & 636 & $49 \cdot 6$ & 844 & $58 \cdot 5$ \\
\hline $7-11$ & 384 & $29 \cdot 9$ & 407 & $28 \cdot 2$ \\
\hline$\geq 12$ & 263 & $20 \cdot 5$ & 192 & $13 \cdot 3$ \\
\hline \multicolumn{5}{|l|}{ Social class ${ }^{*} \dagger$} \\
\hline I-II & 162 & $12 \cdot 5$ & 100 & 6.9 \\
\hline III & 459 & 35.5 & 427 & $29 \cdot 7$ \\
\hline IV-V & 498 & 38.5 & 700 & $48 \cdot 6$ \\
\hline Other & 174 & $13 \cdot 5$ & 212 & $14 \cdot 7$ \\
\hline \multicolumn{5}{|l|}{ BMI $\left(\mathrm{kg} / \mathrm{m}^{2}\right)^{*}$} \\
\hline$<24$ & 278 & 21.5 & 336 & $23 \cdot 2$ \\
\hline $24-25 \cdot 9$ & 340 & $26 \cdot 4$ & 342 & $23 \cdot 6$ \\
\hline $26-28 \cdot 9$ & 403 & $31 \cdot 2$ & 345 & $31 \cdot 2$ \\
\hline$\geq 29$ & 269 & $20 \cdot 8$ & 317 & 21.9 \\
\hline \multicolumn{5}{|l|}{ Smoking status } \\
\hline Non-smoker & 370 & $28 \cdot 6$ & 344 & $23 \cdot 7$ \\
\hline Ex-smoker & 270 & $20 \cdot 9$ & 416 & $28 \cdot 7$ \\
\hline Current smoker & 654 & $50 \cdot 5$ & 691 & $47 \cdot 6$ \\
\hline \multicolumn{5}{|c|}{ Family history of prostate cancerł } \\
\hline No & 1204 & $93 \cdot 0$ & 1423 & $98 \cdot 1$ \\
\hline Yes & 90 & $7 \cdot 0$ & 28 & 1.9 \\
\hline
\end{tabular}

* The sum does not add up to the total because of some missing values.

†I-II: professional, managerial, intermediate; III: skilled occupations; IV-V: partly skilled and unskilled occupations; other: farmers, and other or unknown occupations.

$\ddagger$ In first-degree relatives.

\section{Discussion}

The present study, being one of the largest case-control investigations on diet and prostate cancer to date in a southern European population, shows a positive association between the DII and the risk of prostate cancer with statistically significant risk estimates for the DII expressed as a continuous variable and for men in the third and fourth quartiles of the DII ( $v$. the first quartile). However, there was a levelling of risk across the two highest quartiles. We also observed a reduction in the consumption of healthy food items such as vegetables, fruits and fish, and an increase in the consumption of unhealthy food items such as pork, cheese and sugars, with increasing DII scores. This result supports the hypothesis that men who consume a pro-inflammatory diet are at a higher risk of developing prostate cancer ${ }^{(11)}$.

Various dietary factors exert an array of effects on prostate cancer; some of these are pro-inflammatory (e.g. meat intake $\left.^{(4)}\right)$ and some are anti-inflammatory (e.g. isoflavone (37) $^{(3)}$ and soya ${ }^{(38)}$ intake). The positive association of the DII with the risk of prostate cancer found in the present casecontrol study is of specific interest. One of the possible mechanisms responsible for this association is the effect of the pro-inflammatory diet on systemic inflammation and 
Table 2. Distribution of food groups across quartiles of the dietary inflammatory index (DII) in the study conducted in Italy between 1991 and 2002

(Mean values and standard deviations)

\begin{tabular}{|c|c|c|c|c|c|c|c|c|c|}
\hline \multirow[b]{3}{*}{ Food groups } & \multicolumn{8}{|c|}{ DII quartile (servings/week) } & \multirow[b]{3}{*}{$P^{\star}$} \\
\hline & \multicolumn{2}{|c|}{1} & \multicolumn{2}{|c|}{2} & \multicolumn{2}{|c|}{3} & \multicolumn{2}{|c|}{4} & \\
\hline & Mean & SD & Mean & SD & Mean & SD & Mean & SD & \\
\hline All vegetables & $16 \cdot 21$ & $6 \cdot 30$ & $13 \cdot 17$ & $5 \cdot 12$ & $10 \cdot 67$ & 4.56 & 7.88 & 5.55 & $<0.0001$ \\
\hline Raw vegetables & 11.75 & 5.92 & $9 \cdot 48$ & 4.78 & $7 \cdot 67$ & $4 \cdot 28$ & 5.83 & $5 \cdot 33$ & $<0.0001$ \\
\hline Cooked vegetables & 4.46 & 2.35 & 3.68 & $2 \cdot 18$ & 3.01 & 1.77 & 2.05 & 1.44 & $<0.0001$ \\
\hline Fruits & $23 \cdot 20$ & $10 \cdot 77$ & 18.93 & $9 \cdot 11$ & 14.72 & 7.92 & $10 \cdot 46$ & 7.41 & $<0.0001$ \\
\hline Red meat & $4 \cdot 20$ & $2 \cdot 39$ & 4.50 & $2 \cdot 29$ & 4.51 & $2 \cdot 33$ & $4 \cdot 13$ & $2 \cdot 25$ & 0.57 \\
\hline Pork & 2.45 & 1.93 & $2 \cdot 61$ & 2.02 & $2 \cdot 83$ & $2 \cdot 15$ & $2 \cdot 76$ & 2.29 & 0.002 \\
\hline Poultry & 1.85 & 1.41 & 1.90 & 1.36 & 1.74 & $1 \cdot 26$ & 1.56 & $1 \cdot 29$ & $<0.0001$ \\
\hline Sugars & 34.52 & 37.03 & $42 \cdot 51$ & 37.75 & 49.33 & $49 \cdot 67$ & 48.09 & 47.54 & $<0.0001$ \\
\hline Artificial sweeteners & 1.65 & 6.43 & 1.71 & 7.93 & $1 \cdot 24$ & $5 \cdot 12$ & $1 \cdot 73$ & 7.39 & 0.83 \\
\hline Fish & 2.04 & 1.27 & 1.79 & 0.99 & 1.73 & 1.01 & 1.45 & 1.00 & $<0.0001$ \\
\hline Cheese & 3.87 & $2 \cdot 12$ & 4.68 & $2 \cdot 81$ & 4.72 & 2.93 & 4.86 & $3 \cdot 3$ & $<0.0001$ \\
\hline Milk & 6.09 & $6 \cdot 12$ & $6 \cdot 17$ & $6 \cdot 17$ & $5 \cdot 76$ & $6 \cdot 58$ & $6 \cdot 10$ & $6 \cdot 90$ & 0.71 \\
\hline Bread & $19 \cdot 21$ & $10 \cdot 50$ & $22 \cdot 83$ & $12 \cdot 40$ & 24.38 & $14 \cdot 22$ & $23 \cdot 12$ & 14.79 & $<0.0001$ \\
\hline
\end{tabular}

${ }^{\star} P$ values were obtained from ANOVA.

insulin resistance ${ }^{(39,40)}$. Consumption of foods such as meat and butter have been shown to increase systemic inflammation by increasing levels of high-sensitive C-reactive protein, E-selection and soluble vascular cell adhesion molecule- ${ }^{(39)}$, which then are responsible for increasing insulin resistance ${ }^{(40)}$. Increasing insulin resistance leads to hyperinsulinaemia, which has been demonstrated to play a role in the development of prostate cancer by inhibiting apoptosis, stimulating cell proliferation ${ }^{(41)}$ and influencing the insulin-like growth factor axis with consequent alterations in sex hormone metabolism. Along this line, a diet high in glycaemic load has been related to an increased risk of prostate cancer $^{(42)}$. Also, a diet rich in pro-inflammatory constituents, such as saturated fat, causes proliferation, inflammation and oxidative stress that can lead to benign prostatic hyperplasia, prostatitis, and possibly cancer of the prostate ${ }^{(43)}$.

The influence of diet on prostate cancer is difficult to evaluate, and challenges in dietary exposure assessment are greatest in case-control studies. A human diet consists of both proinflammatory and anti-inflammatory foods, nutrients and other food constituents; thus, the DII score, which takes into account both categories of dietary exposure, more accurately reflects the relationship of diet with the risk of cancer than individual nutrients. Although (hospital-based) case-control studies are thought to be more susceptible to selection and information bias than cohort studies ${ }^{(36)}$, several factors argue in support of the validity of the present investigation. Dietary information was elicited using a valid and reproducible FFQ $^{(32-34)}$, which was comprehensive enough to allow adjustment for total energy intake. This was administered to cases and controls by the same interviewers under similar conditions. To minimise any recall bias due to the onset or treatment of the disease, individuals were asked about food intake in the 2 years before the interview. The potential bias in the recall of food intake, however, should be limited in Italy, because the diet and prostate cancer issue has not had widespread interest. Also, controls were selected from the same hospital system as the cases and therefore are more likely to be subject to similar sorts of recall bias. Indeed, hospital-derived controls should be less prone to information bias than population ones ${ }^{(36)}$. Furthermore, the cases and controls were selected from the same catchment areas, the participation rate was high, particular attention was paid to exclude from the control group diseases potentially linked to the diet and long-term dietary modifications, and major confounding factors of prostate cancer were accounted for.

Table 3. Odds ratios of prostate cancer according to quartiles of the dietary inflammatory index (DII), among 1294 cases and 1451 controls, in the study conducted in Italy between 1991 and 2002

(Odds ratios and $95 \%$ confidence intervals)

\begin{tabular}{|c|c|c|c|c|c|c|c|}
\hline & \multicolumn{4}{|c|}{ DII quartile cut-points (median) } & $P_{\text {trend }}$ & $\mathrm{OR}^{*}$ & $95 \% \mathrm{Cl}$ \\
\hline Cases $(n)$ & 279 & 337 & 363 & 315 & & & \\
\hline Age-adjusted OR & $1 \dagger$ & 1.05 & 1.04 & 0.89 & 0.22 & 1.00 & $0.92,1.01$ \\
\hline $95 \% \mathrm{Cl}$ & & $0.84,1.31$ & $0.84,1.30$ & $0.71,1.11$ & & & \\
\hline Multivariable OR $\ddagger$ & $1 \dagger$ & $\begin{array}{c}1.19 \\
0.941 .50\end{array}$ & $\begin{array}{c}1.32 \\
1.031 .69\end{array}$ & $\begin{array}{c}1.33 \\
1.011 .76\end{array}$ & 0.04 & 1.06 & $1 \cdot 00,1 \cdot 13$ \\
\hline
\end{tabular}

${ }^{\star}$ Continuous OR for a one-unit increment in the DII, corresponding to approximately $7 \%$ of its global range.

† Reference category.

$\ddagger$ Adjusted for age, study centre, BMI, years of education, social class, smoking status, family history of prostate cancer, and total energy intake. 
It also should be made clear that not all of the forty-five food parameters mentioned in the study on the development of the DII ${ }^{(25)}$ were used for the calculation of the DII. For the current DII calculation, we used the data from thirty-two food parameters, and most of these had article weights of $>236$ (indicating optimal confidence in the evidence base). However, we do understand that about half of the food parameters had article weights below the median level of 236. Many of these food parameters were not studied extensively in the current scenario. These include foods such as rosemary, thyme and oregano that are consumed in relatively small amounts. Hence, we feel that there is no immediate need to update the literature review; however, we may consider updating in the future.

The regionally representative database was created to include dietary consumption of the forty-five food parameters from eleven countries. These eleven countries were selected from different regions of the world, in order to obtain a wide spectrum of consumption of these food parameters. The countries included are the USA, Mexico, England, Denmark, India, Australia, New Zealand, Bahrain, Scotland, South Korea and Japan ${ }^{(25)}$. So, the mean values of the food parameters from this database should be representative of the average consumption of these parameters across the world. Notwithstanding the limitations of case-control studies in general, we believe that our findings of a positive association of the DII with the risk of prostate cancer are plausible and could be related to immune and hormonal factors $^{(41,43,44)}$.

In conclusion, this uniquely large study on prostate cancer and the DII conducted in a southern European population indicates a possible role of diet in prostate cancer risk through the process of inflammation. However, confirmatory results from other studies conducted in different populations with different study designs are required to truly establish this association.

\section{Acknowledgements}

The present study was supported by the Italian Foundation for Cancer Research (FIRC) and the IRCCS Centro di Riferimento Oncologico, Aviano (Ricerca Corrente 2009), Italy. J. R. H. was supported by an Established Investigator Award in Cancer Prevention and Control from the Cancer Training Branch of the National Cancer Institute (K05 CA136975).

The authors' contributions are as follows: N. S. computed the dietary inflammatory index, contributed to the design of the analyses, led statistical analyses, interpreted results and wrote initial drafts of the manuscript; C. B. helped with the design and implementation of the study, data management, consulted on statistical analyses, interpretation of results of statistical analyses, and writing and revising of the manuscript; A. Z., M. M., D. S. and C. L. V. contributed to the study design, data collection, data interpretation, and revision of the manuscript; J. R. H. invented the dietary inflammatory index, helped to design statistical analyses, interpreting results of statistical analyses, and writing of the manuscript.

The authors declare that there are no conflicts of interest.

\section{References}

1. Touvier M, Fezeu L, Ahluwalia N, et al. (2013) Association between prediagnostic biomarkers of inflammation and endothelial function and cancer risk: a nested case-control study. Am J Epidemiol 177, 3-13.

2. Keibel A, Singh V \& Sharma MC (2009) Inflammation, microenvironment, and the immune system in cancer progression. Curr Pharm Des 15, 1949-1955.

3. Kopp TI, Friis S, Christensen J, et al. (2013) Polymorphisms in genes related to inflammation, NSAID use, and the risk of prostate cancer among Danish men. Cancer Genet 206, 266-278.

4. Cross AJ, Peters U, Kirsh VA, et al. (2005) A prospective study of meat and meat mutagens and prostate cancer risk. Cancer Res 65, 11779-11784.

5. Nakai Y \& Nonomura N (2013) Inflammation and prostate carcinogenesis. Int J Urol 20, 150-160.

6. Pan MH, Lai CS, Dushenkov S, et al. (2009) Modulation of inflammatory genes by natural dietary bioactive compounds. J Agric Food Chem 57, 4467-4477.

7. Coussens LM \& Werb Z (2002) Inflammation and cancer. Nature 420, 860-867.

8. Philip M, Rowley DA \& Schreiber H (2004) Inflammation as a tumor promoter in cancer induction. Semin Cancer Biol 14, 433-439.

9. Kim Y, Jeon $\mathrm{Y}$, Lee $\mathrm{H}$, et al. (2013) The prostate cancer patient had higher $\mathrm{C}$-reactive protein than $\mathrm{BPH}$ patient. Korean J Urol 54, 85-88.

10. Tindall EA, Severi G, Hoang HN, et al. (2012) Interleukin-6 promoter variants, prostate cancer risk, and survival. Prostate 72, 1701-1707.

11. Kazma R, Mefford JA, Cheng I, et al. (2012) Association of the innate immunity and inflammation pathway with advanced prostate cancer risk. PLOS ONE 7, e51680.

12. Kolonel LN (1996) Nutrition and prostate cancer. Cancer Causes Control 7, 83-94.

13. Gronberg H (2003) Prostate cancer epidemiology. Lancet 361, 859-864.

14. World Cancer Research Fund/American Institute for Cancer Research (2007) Second Expert Report: Food, Nutrition, Physical Activity and the Prevention of Cancer: A Global Perspective. Washington, DC: American Institute for Cancer Research.

15. Giovannucci E, Rimm EB, Colditz GA, et al. (1993) A prospective study of dietary fat and risk of prostate cancer. J Natl Cancer Inst 85, 1571-1579.

16. Talamini R, Franceschi S, La Vecchia C, et al. (1992) Diet and prostatic cancer: a case-control study in northern Italy. Nutr Cancer 18, 277-286.

17. La Vecchia C, Negri E, D’Avanzo B, et al. (1991) Dairy products and the risk of prostatic cancer. Oncology 48, 406-410.

18. Talamini R, La Vecchia C, Decarli A, et al. (1986) Nutrition, social factors and prostatic cancer in a Northern Italian population. BrJ Cancer 53, 817-821.

19. Chan JM, Giovannucci E, Andersson SO, et al. (1998) Dairy products, calcium, phosphorous, vitamin $\mathrm{D}$, and risk of prostate cancer (Sweden). Cancer Causes Control 9, 559-566.

20. Le Marchand L, Kolonel LN, Wilkens LR, et al. (1994) Animal fat consumption and prostate cancer: a prospective study in Hawaii. Epidemiology 5, 276-282.

21. Giovannucci E, Ascherio A, Rimm EB, et al. (1995) Intake of carotenoids and retinol in relation to risk of prostate cancer. J Natl Cancer Inst 87, 1767-1776. 
22. Jain MG, Hislop GT, Howe GR, et al. (1999) Plant foods, antioxidants, and prostate cancer risk: findings from casecontrol studies in Canada. Nutr Cancer 34, 173-184.

23. Schuurman AG, Goldbohm RA, Dorant E, et al. (1998) Vegetable and fruit consumption and prostate cancer risk: a cohort study in The Netherlands. Cancer Epidemiol Biomarkers Prev 7, 673-680.

24. Hsing AW, McLaughlin JK, Schuman LM, et al. (1990) Diet, tobacco use, and fatal prostate cancer: results from the Lutheran Brotherhood Cohort Study. Cancer Res 50, 6836-6840.

25. Shivappa N, Steck SE, Hurley TG, et al. (2013) Designing and developing a literature-derived, population-based dietary inflammatory index. Public Health Nutr 17, 1689-1696.

26. Shivappa N, Steck SE, Hurley TG, et al. (2013) A populationbased dietary inflammatory index predicts levels of C-reactive protein in the Seasonal Variation of Blood Cholesterol Study (SEASONS). Public Health Nutr 17, 1825-1833.

27. Wirth MD, Burch J, Shivappa N, et al. (2014) Association of a dietary inflammatory index with inflammatory indices and metabolic syndrome among police officers. J Occup Environ Med 56, 986-989.

28. Wood L, Shivappa N, Berthon BS, et al. (2014) Dietary inflammatory index is related to asthma risk, lung function and systemic inflammation in asthma. Clin Exp Allergy (Epublication ahead of print version 8 April 2014).

29. Shivappa N, Prizment AE, Blair CK, et al. (2014) Dietary inflammatory index (DII) and risk of colorectal cancer in Iowa Women's Health Study. Cancer Epidemiol Biomarkers Prev 23, 2383-2392.

30. Bosetti C, Micelotta S, Dal Maso L, et al. (2004) Food groups and risk of prostate cancer in Italy. Int $J$ Cancer 110, 424-428.

31. Gnagnarella P, Parpinel M, Salvini S, et al. (2004) The update of the Italian food composition database. J Food Comp Analysis 17, 509-522.

32. Decarli A, Franceschi S, Ferraroni M, et al. (1996) Validation of a food-frequency questionnaire to assess dietary intakes in cancer studies in Italy. Results for specific nutrients. Ann Epidemiol 6, 110-118.
33. Franceschi S, Negri E, Salvini S, et al. (1993) Reproducibility of an Italian food frequency questionnaire for cancer studies: results for specific food items. Eur J Cancer 29A, 2298-2305.

34. Franceschi S, Barbone F, Negri E, et al. (1995) Reproducibility of an Italian food frequency questionnaire for cancer studies. Results for specific nutrients. Ann Epidemiol 5, 69-75.

35. Danesh J, Whincup P, Walker M, et al. (2000) Low grade inflammation and coronary heart disease: prospective study and updated meta-analyses. BMJ 321, 199-204.

36. Breslow NE \& Day N (1980) Statistical methods in cancer research. Volume 1 - the analysis of case-control studies. IARC Sci Publ 32, 5-338.

37. Travis RC, Spencer EA, Allen NE, et al. (2009) Plasma phytooestrogens and prostate cancer in the European Prospective Investigation into Cancer and Nutrition. Br J Cancer 100, $1817-1823$.

38. Hebert JR, Hurley TG, Olendzki B, et al. (1998) Nutritional and socioeconomic factors in relation to prostate cancer mortality: a cross-national study. J Natl Cancer Inst 90, $1637-1647$.

39. Esmaillzadeh A, Kimiagar M, Mehrabi Y, et al. (2007) Dietary patterns and markers of systemic inflammation among Iranian women. J Nutr 137, 992-998.

40. Festa A, D'Agostino R, Howard G, et al. (2000) Chronic subclinical inflammation as part of the insulin resistance syndrome: the Insulin Resistance Atherosclerosis Study (IRAS). Circulation 102, 42-47.

41. Kaaks R \& Lukanova A (2001) Energy balance and cancer: the role of insulin and insulin-like growth factor-I. Proc Nutr Soc 60, 91-106.

42. Augustin LS, Galeone C, Dal Maso L, et al. (2004) Glycemic index, glycemic load and risk of prostate cancer. Int J Cancer 112, 446-450.

43. Vykhovanets EV, Shankar E, Vykhovanets OV, et al. (2011) High-fat diet increases NF- $\mathrm{B}$ signaling in the prostate of reporter mice. Prostate $\mathbf{7 1}, 147-156$.

44. Pandey M \& Gupta S (2009) Green tea and prostate cancer: from bench to clinic. Front Biosci (Elite Ed) 1, 13-25. 